\title{
HYPORETINOLEMIE ET SOUS-NUTRITION CHEZ LES ENFANTS DE 24 A 59 MOIS ET LES FEMMES EN AGE DE PROCURER DANS DEUX MILIEUX RURAL ET URBAIN DU MALI .
}

\section{HYPORETINOLEMIE AND MALNUTRITION AT THE CHILDREN OF 24 - 59 MONTHS AND THE WOMEN IN AGE TO PROCREATE IN TWO RURAL AND URBAIN AREAS OF MALI.}

AG IKNANE A ${ }^{1}$, KASSOGUE K² , DJANGO DM ${ }^{3}$, DIAWARA A ${ }^{4}$, THIERO TA ${ }^{5}$, KONATE K ${ }^{6}$, SANGHO H7,TRAORE AK ${ }^{8}$,

${ }^{1}$ Maître assistant en santé publique, Chef du service Nutrition, Institut National de Recherche en Santé Publique, Bamako, Mali

2 Médecin généraliste, Bamako, Mali

3 Maître assistant en anesthésie réanimation, FMPOS, Bamako, Mali

${ }^{4}$ Maître de conférences en santé publique, Directeur de l'Agence Nationale d'Evaluation des Hôpitaux (ANEH) - Bamako, Mali

${ }_{5}^{5}$ Biologiste, gestionnaire des services de santé, chef du département de Diagnostic et recherche biomédicale, Institut National de Recherche en Santé Publique, Bamako

${ }^{6}$ Assistante médicale, Institut National de Recherche en santé Publique (INRSP), Bamako, Mali*

${ }^{6}$ Maître de conférence en santé publique à la FMPOS, Directeur du CREDOS- Bamako, Mali

${ }^{7}$ Maître de conférences agrégé en médecine interne, FMPOS, Bamako, Mali

Remerciements: Nous remercions Heller Keller International pour le financement de la présente etude.

Résumé

Une étude transversale stratifiée par sondage en grappe s'est déroulée du 30 janvier au 16 mars 2006 dans deux régions du Mali : Koulikoro (zone rurale) et le district de Bamako (zone urbaine). Elle avait porté sur un échantillon de 692 enfants de 24 à 59 mois et 803 femmes en âge de procréer. Le dosage du rétinol sérique avait été réalisé pour déterminer la prévalence de l'hyporétinolémie chez les enfants de 24 à 59 mois et les femmes en âge de procréer et ses liens avec la malnutrition ont été analysés. L'étude a montré que $78,2 \%$ des enfants de 24 à 59 mois ruraux présentaient une hyoporétinolémie significativement plus prononcée qu'en milieu urbain avec $17 \%$. Chez les femmes aussi, les rurales sont plus touchées par cette carence avec 50 $\%$ contre $29,2 \%$ en milieu urbain. Seul chez les enfants ruraux, il a été trouvé un lien entre l'émaciation et l'hyporetinolemie $(p=0,000)$. Par contre, aucun lien n'a été observé entre l'hyoporétinolémie et les autres formes de malnutrition ni chez l'enfant ni chez les femmes en âge de procréer $(p<0,05)$.

\section{Summary}

A transverse study stratified by poll in cluster unfolded itself of January 30 to March 162006 in two regions of the Mali: Koulikoro (rural zone) and the district of Bamako (urban zone). He concerned a sample of 692 children of 24 to 59 month and 803 women in age to procreate. The dosage of the blood retinol had been realized to determine the prevalency of the deficiency in vitamin A with the children of $24-59$ month and the women in age to procreate and its links with the malnutrition were analyzed. The study showed that $78,2 \%$ of children of 24 to 59 rural month presented a deficiency in vitamin A significantly more pronounced than in urban environment with $17 \%$. With the women also, the rural ones more are touched by this lack with $50 \%$ in rural environment and $29,2 \%$ in urban environment. Only the relation between the emaciation with the rural children and the deficiency in vitamin $A$ was shown $(p=0,000)$. On the other hand, no link was observed between the deficiency in vitamin $A$ and the others form of malnutrition with the child and the chronic energetic deficiency with the women in age to procreate $(p<0,05)$.

Keys words: Hyporetinolemia, malnutrition, vitamin A deficiency.

\section{INTRODUCTION}

La vitamine A est un micronutriment essentiel au maintien des fonctions physiologiques normales, la vision, les réponses immunitaires et la croissance [1]. Sa carence ou de ses précurseurs fait apparaître des troubles de plus en plus graves de ses fonctions [2]. C'est une affection grave, en particulier de la première enfance, qui se traduit par un retard de développement, une gravité accrue des infections, et plus tardivement, par une atteinte oculaire caractéristique appelée xérophtalmie [2]. L'avitaminose A est étroitement liée à l'augmentation de la morbidité et de la mortalité infantile, au risque de diarrhée et d'infections pulmonaires et s'accompagne presque toujours d'une malnutrition [3], obstacle à l'absorption et au stockage de la vitamine $A$. Ainsi, l'infection exacerbe la carence et affecte la réponse immunitaire [4]. Selon l'OMS, 34 pays dans le monde connaissent des déficits en vitamine $A$, dont 18 sont d'Afrique de l'ouest [7]. Dans le monde, plus de 13 millions d'enfants présentent des signes ophtalmiques liés à cette carence [6]. En Afrique, près de 18 millions d'enfants est à risque dont 1,3 millions souffrent de xérophtalmie [8]. Au Mali, une étude réalisée en 2001 [9] montre que la prévalence ajustée de cécité crépusculaire chez les femmes était de $5.8 \%$ ce qui est supérieur au seuil de $5 \%$ établi pour la définition de la carence en vitamine $A$ en tant que problème de santé publique. Cette prévalence est inégalement répartie entre les régions du pays, avec $6.3 \%$ pour la région de Koulikoro et $2.9 \%$ pour Bamako. Au Mali, environ 16353 décès d'enfants de 6 à 59 mois sont attribuables à la carence en vitamine $A[9,10]$, ce qui en fait un problème de santé publique $[10,9]$. La présente étude avait pour objectif d'évaluer le statut vitaminique $A$ des femmes en âge de procréer et des enfants de 24 à 59 mois dans le district de Bamako et la région de Koulikoro à travers le dosage du rétinol sérique et déterminer son lien avec la malnutrition. 


\section{MATERIELS ET METHODES.}

L'étude était de type transversale stratifiée avec un sondage en grappe à trois degré à l'intérieur de chaque strate qui s'est déroulée 30 janvier au 16 mars 2006. La strate urbaine, constituée par le district de Bamako avec une population de 1.016167 habitants [11] repartie entre 6 communes et la strate rurale constituée par la région de Koulikoro avec une population de 1516486 habitants repartie entre 7 cercles constituaient le cadre de l'étude. La population étudiée était constituée par les enfants de 24 à 59 mois (440 à Koulikoro et 363 à Bamako) et les femmes en âge de procréer (445 à Koulikoro et 247 à Bamako).

Le nombre de ménages retenu dans l'étude a été calculé à partir de la formule habituelle (nombre de ménages = Nombre de femmes / (taux de réponse * nombre de femmes par ménage). Un taux de réponse de $90 \%$ utilisé par l'EDSM III avait été retenu [9]. Le nombre de femmes et d'enfants avait été tiré en tenant compte du taux de prévalence du déficit en vitamine $A$ qui oscille entre $30 \%$ et $50 \%$. La taille de l'échantillon a été déterminée par la formule usuelle de Schwartz. Des prélèvements de sang veineux chez les femmes et les enfants avaient été effectués pour le dosage du rétinol sérique à partir du Laboratoire Muraz de Bobo-Dioulasso. Des mesures anthropométriques (poids, taille, âge, sexe,) de la population étudiée ont complété la collecte des données. Une valeur de rétinol sérique $\geq 0,70$ $\mu \mathrm{mol} / \mathrm{l}$, était considéré comme signe de carence. Les données anthropométriques ont été normalisées à partir de la table standard OMS de décembre 2005 et un seuil $<-2$ ET avait été retenu pour définir la malnutrition chez l'enfant. Les données normalisées ont été analysées à l'aide du logiciel SPSS.12.0. Le test du $\mathrm{Chi}^{2}$ de Pearson avait été utilisé pour la comparaison des moyennes. Au plan éthique, un consentement préalable était obtenu et les interviews s'étaient déroulées dans le respect de l'intimité des ménages. De plus, la prise en charge des sujets malades avait été assurée.

\section{RESULTATS}

L'étude avait concerné 692 enfants de 24 à 59 mois dont 440 du milieu rural (Koulikoro), 363 du milieu urbain (Bamako) et 803 femmes en âge de procréer dont 445 à Koulikoro et 247 à Bamako soit au total 1495 personnes. L'Hyoporétinolemie de 78,2\% était significativement plus importante chez les enfants ruraux que ceux du milieu urbain avec $17 \%(p=0,0001)$. Cette carence en vitamine A était 4,5 fois plus élevée en milieu rural qu'en milieu urbain. L'âge des enfants n'avait pas d'influence sur le niveau de prévalence du rétinol sanguin $(p<0,05)$. En milieu rural, la prévalence de la rétinolémie était à plus de $50 \%$ dans tous les cercles avec des pics plus importants à Koulikoro, Kolokani et Kati qui ont enregistré des taux de prévalence les plus élevés avec respectivement $94,2 \%, 87,7 \%$ et $85,6 \%$ contrairement à ceux de Doïla et Kangaba avec $52,5 \%$ et $57,1 \%$. En milieu urbain par contre, à l'exception des commune 1 $(38,7 \%)$ et $3(6,3 \%)$, les niveaux de prévalence étaient plus faibles, mais restaient autour de 15 à $18 \%$. $(p<0,05)$.
Chez les femmes en âge de procréer, la prévalence de la rétinolemie variait de $50 \%$ en milieu rural à $29,2 \%$ en milieu urbain. Les tranches d'âge 20 - 24 ans et $25-29$ ans étaient les plus touchées dans les deux milieux. La relation entre l'hyporétinolémie et la malnutrition montre un lien significatif avec l'émaciation en milieu rural ( $p=0,0001)$, avec $90 \%$ d'enfants carencés en vitamine A émaciés. En milieu urbain par contre, aucune relation n'est observée. II en est de même pour le retard de croissance et l'insuffisance pondérale aussi bien en milieu rural qu'urbain avec respectivement 82,1\% versus $35,5 \%$ pour le milieu urbain et $84,8 \%$ contre 13,6 $\%$ pour le milieu urbain. Chez les femmes, c'était 48,3\% d'entre elles qui présentaient un déficit énergétique chronique associé au déficit en vitamine $A$ en milieu rural contre $25 \%$ en milieu urbain sans différence significative.

\section{DISCUSSION}

La prévalence de l'émaciation chez les enfants est comparable dans les deux milieux $(4,5 \pm 0,65 \%$ en milieu rural contre $4 \pm 0,63 \%$ en milieu urbain) sans différence significative selon le sexe. Les enfants de 24 à 35 mois sont légèrement plus touchés que les autres dans les deux milieux. Par contre, le risque d'émaciation semble plus élevé en milieu urbain qu'en milieu rural (30,6\% contre 19,6\%).

Le retard de croissance est trois fois plus élevé en milieu rural qu'en milieu urbain avec respectivement 24,4 \pm $0,93 \%$ et $6,9 \pm 0,87 \%$. Là aussi, il n'y a pas de différence selon le sexe et la tranche d'âge, même si les 24 à 35 mois semblent plus touchés dans les deux milieux. Le risque de retard de croissance par contre est plus élevé en milieu urbain qu'en milieu rural (30,6\% contre 19,6\%). Ici aussi, il n'y a pas de différence selon le sexe et la tranche d'âge, même si les 24 à 35 mois semblent plus touchés dans les deux milieux $(20,6 \%$ contre $14,1 \%$ en milieu urbain).

Une enquête nationale récente [12] trouve $14 \%$ d'émaciation à Koulikoro contre $4,5 \%$ dans notre étude contre $12,4 \%$ à Bamako comparée à 3,6 \% pour notre étude. Chez les femmes en âge de procréer, le déficit énergétique chronique était de $13,2 \% \pm 0,65$ en milieu rural contre $6,6 \% \pm 0,61$ en milieu urbain, soit deux fois plus, mais reste comparable.

Le déficit énergétique chronique frappait prioritairement les femmes jeunes de moins de 30 ans dans les deux strates. II en est de même pour le risque de déficit énergétique chronique plus prononcé en milieu rural. Parmi les femmes en âge de procréer présentant ayant un déficit énergétique chronique, 47,5\% d'entre elles résident en milieu rural sont carencées en vitamine $A$ contre $24 \%$ pour le milieu urbain.

\section{Conclusion et recommandations}

Notre étude, première du genre au Mali à déterminer la prévalence de la rétinolémie sanguine, le niveau de prévalence obtenu reste très élevé en milieu rural avec un lien significatif avec l'émaciation chez les enfants prononcé en milieu rural. L'association forte entre carence en vitamine $A$ et émaciation notamment en milieu rural, ainsi que l'association forte entre émaciation 
et insuffisance pondérale en milieu rural ont démontré la nécessité de renforcer les actions de lutte contre toutes les carences nutritionnelles notamment en milieu rural. La multisectorialité de la lutte contre la malnutrition, le pragmatisme et la souplesse dans les interventions devraient être privilégiés si l'on voudrait aboutir à des résultats à moyen ou long terme dans ce domaine. Une étude de prévalence de la rétinolémie au niveau nationale et ses liens avec les autres carences nutritionnelles aideraient mieux les politiques et programmes nationaux en matière de nutrition.

\section{REFERENCES BIBLIOGRAPHIQUES}

1. SOMMER A.: La carence en vitamine $A$ et ses conséquences :guide pratique de dépistage et de la lutte contre le déficit en vitamine A. 3ème édit. ;1995.

2. AMEDEE MANESME. 0 ; DE MAYER. E. Le déficit en vitamine A. Edition INSERM. ORSTOM ; Paris, 1989.

3. O.M.S.: Supplément en vitamine A. Guide pour leur emploie dans le traitement et la prévention de la carence en vitamine $A$ et de la xérophtalmie. Genève 1989, O.M.S./FISE/IVAG.

4. AG Bendech. M ; Les pratiques prometteuses et les leçons apprises dans la lutte contre la carence en vitamine A dans les pays de l'Afrique sub saharienne. Basics; 2000

5. Donald S. MC Laren. Martin Frigg. Manuel sight and life sur les troubles dus à la carence en vitamine A. 2éme édit ; 2002 ; Suisse.

6. Centre International de nutrition (CIN) ; Les grands enjeux des stratégies nutritionnelles - préventions carences spécifiques en micronutriments. FAO/OMS, 1992, ROME ; P1-47.

\begin{tabular}{|l|c|c|c|c|}
\hline \multirow{2}{*}{$\begin{array}{l}\text { Principales } \\
\text { carences } \\
\text { nutritionnelles }\end{array}$} & $\begin{array}{c}\text { Enflieu } \\
\text { rural } \\
\mathrm{n}=440 \\
(\%)\end{array}$ & $\begin{array}{c}\text { Milieu } \\
\text { urbain } \\
\mathrm{n}=363 \\
(\%)\end{array}$ & $\begin{array}{c}\text { Milieu } \\
\text { rural } \\
\mathrm{n}=445 \\
(\%)\end{array}$ & $\begin{array}{c}\text { Milieu } \\
\text { urbain } \\
\mathrm{n}=247 \\
(\%)\end{array}$ \\
\hline $\begin{array}{l}\text { Carences } \\
\text { vitamine A }\end{array}$ & 78,2 & 17,0 & 50,0 & 29,2 \\
\hline Emaciation / DEC & 4,5 & 3,6 & 13,2 & 6,6 \\
\hline $\begin{array}{l}\text { Retard de } \\
\text { croissance }\end{array}$ & 24,0 & 6,9 & & \\
\hline $\begin{array}{l}\text { Insuffisance } \\
\text { pondérale }\end{array}$ & 18,4 & 8,9 & & \\
\hline
\end{tabular}

Tableau I; Hyporetinolémie et malnutrition chez les enfants de 24 - 59 mois et les femmes en âge de procréer
7. Centre Nationale de Nutrition (CNN). Analyses profiles de la situation nutritionnelle au Burkina Faso. Ministère de la santé. 2001.

8. Centre Pasteur du Cameroun (CPC). Appui à la lutte contre la carence en vitamine A. 2003; Yaoundé (Cameroun).

9. MALI. Enquête Démographique et de Santé Mali (EDSM III, 2001), Ministère de la Santé/Cellule de planification et de statistique (CPS)/Direction Nationale de la Statistique et de l'informatique (DNSI), Macro International, juin 2002, $450 \mathrm{p}$.

10. HELEN KELLER INTERNATIONAL; La cécité crépusculaire chez les mères maliennes est-elle un indicateur de carence en vitamine A dans la population malienne, 2003.

11. DNSI, Projection de la population à partir du RGPH de 1998 jusqu'en 2012

12. MALI. Enquête Démographique et de Santé Mali (EDSM IV, 2006) Ministère de la Santé/Cellule de planification et de statistique (CPS)/Direction Nationale de la Statistique et de l'informatique (DNSI), Macro International, Décembre 2007, 494 p.

13. SOMMER A et al. Increased mortality in children with mild vitamin A deficiency. Lancet, 1993; ii: 585-588.

14. AG IKNANE A- CISSE A T, FOFANA A, TRAORE $A$, KASSOGUE $K$. Enquête de base sur la prévalence des carences en vitamine $A$ et en fer dans le district de Bamako et la région de Koulikoro, MS, HKI, Août 2007.

15. AGUAYP VM, KONE D, WALTENSPERGER K, CISSE A: La cécité crépusculaire chez les mères maliennes est- elle un indicateur de carence en vitamine $A$ dans la population malienne? Mai/juin, 2003.

\begin{tabular}{|l|c|c|c|c|c|c|}
\hline $\begin{array}{l}\text { Principales } \\
\text { carences } \\
\text { nutritionnel } \\
\text { les }\end{array}$ & \multicolumn{3}{|c|}{\begin{tabular}{|} 
Enfants de 24-59 mois \\
n=348
\end{tabular}} & \multicolumn{3}{|c|}{$\begin{array}{c}\text { Femmes en âge de } \\
\text { procréer } \\
\text { n=42 }\end{array}$} \\
\cline { 2 - 7 } & $\begin{array}{c}\text { Milieu } \\
\text { rural (\%) }\end{array}$ & $p$ & $\begin{array}{c}\text { Milieu } \\
\text { urbain } \\
(\%)\end{array}$ & $\begin{array}{c}\text { Milieu } \\
\text { rural (\%) }\end{array}$ & $p$ & $\begin{array}{c}\text { Milieu } \\
\text { urbain } \\
(\%)\end{array}$ \\
\hline $\begin{array}{l}\text { Emaciation } \\
\text { / DEC }\end{array}$ & 90,0 & 0,150 & 0,0 & 48,3 & 0,953 & 25,0 \\
\hline $\begin{array}{l}\text { Retard de } \\
\text { croissance }\end{array}$ & 82,1 & 0,166 & 35,3 & & & \\
\hline $\begin{array}{l}\text { Insuffisanc } \\
\text { e } \\
\text { pondérale }\end{array}$ & 84,8 & 0,075 & 13,6 & & & \\
\hline
\end{tabular}

Tableau 2: Lien entre hyporétinolémie et la malnutrition chez les enfants et les femmes en âge de procréer 